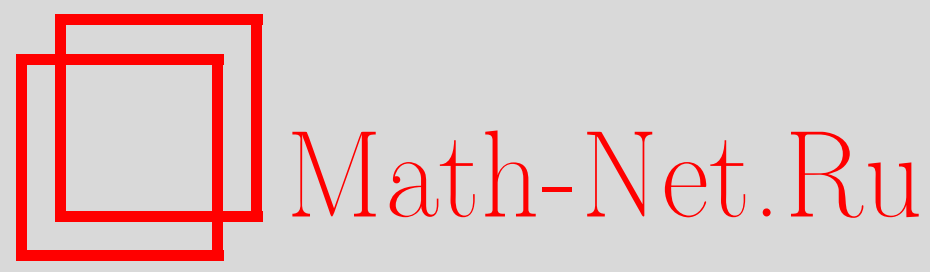

В. И. Овчинников, Когерентно ядерные операторы в парах гильбертовых пространств, Матем. заметки, 1998, том 63, выпуск 6, 866-872

DOI: https://doi.org/10.4213/mzm1357

Использование Общероссийского математического портала Math-Net.Ru подразумевает, что вы прочитали и согласны с пользовательским соглашением http://www . mathnet.ru/rus/agreement

Параметры загрузки:

IP : 54.147 .182 .235

26 апреля 2023 г., 16:43:22

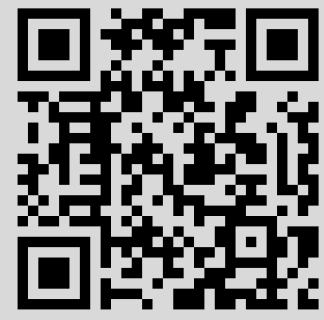




\section{КОГЕРЕНТНО ЯДЕРНЫЕ ОПЕРАТОРЫ \\ В ПАРАХ ГИЛЬБЕРТОВЫХ ПРОСТРАНСТВ}

\section{В.И. Овчинников}

В статье приведено доказательство того, что идеал ядерньх операторов, действующих в паре гильбертовых пространств, совпадает с идеалом когерентно ядерных операторов. Получена новая формула для $K$-функционала в паре алгебр всех ограниченных линейных операторов, действующих в гильбертовых пространствах, образующих пару, а также новые интерполяционные теоремы для ядерных операторов.

Библиография:: 6 названий.

Идеалы операторов, действуюших в банаховых парах, играют важную роль в методе орбит построения и изучения интерполяционных пространств (см. [1]). Среди них идеал когерентно ядерных операторов занимает особое место, поскольку является в определенном смысле минимальным. Напомним, что оператор $T$, действующий из банаховой пары $\left\{X_{0}, X_{1}\right\}$ в банахову пару $\left\{Y_{0}, Y_{1}\right\}$, называется когерентно ядерным, если существуют $x_{j}^{*} \in\left(X_{0}+X_{1}\right)^{*}$ и $y_{j} \in Y_{0} \cap Y_{1}$, где $j \in \mathbb{Z}$, такие, что для всех $x \in X_{0}+X_{1}$

$$
T(x)=\sum_{j \in \mathbb{Z}} x_{j}^{*}(x) y_{j}=\sum_{j \in \mathbb{Z}}\left(y_{j} \otimes x_{j}^{*}\right)(x),
$$

при этом

$$
\sum_{j \in \mathbb{Z}}\left\|x_{j}^{*}\right\|_{X_{0}^{*}}\left\|y_{j}\right\|_{Y_{0}}<\infty, \quad \sum_{j \in \mathbb{Z}}\left\|x_{j}^{*}\right\|_{X_{1}^{*}}\left\|y_{j}\right\|_{Y_{1}}<\infty .
$$

Тем самым, оператор $T$ является ядерным из $X_{0}$ в $Y_{0}$ и из $X_{1}$ в $Y_{1}$, причем разложение в абсолютно сходяшийся ряд одномерных операторов происходит одновременно в обоих пространствах пары.

Через $\operatorname{Cohn}\left(\left\{X_{0}, X_{1}\right\} \rightarrow\left\{Y_{0}, Y_{1}\right\}\right)$ обозначим пространство всех когерентно ядерных операторов.

Если $H$ и $G$ - гильбертовы пространства, то через $S_{p}(H \rightarrow G)$ будем обозначать пространство операторов $T: H \rightarrow G$ таких, что $\left(T^{*} T\right)^{p / 2}$ - ядерньй оператор в пространстве $H$. При $p=1$ получаем пространство всех ядерных операторов, при $p=2-$ пространство всех операторов Гильберта-Шмидта. Введем норму в $S_{p}(H \rightarrow G)$

$$
\|T\|_{S_{p}(H \rightarrow G)}=\left(\operatorname{tr}\left(T^{*} T\right)^{p / 2}\right)^{1 / p}, \quad 1 \leqslant p<\infty .
$$

Работа выполнена при частичной поддержке Международного научного фонда и Российского правительства, грант JD 7100. 
Для краткости будем писать $S_{p}(H)$ вместо $S_{p}(H \rightarrow H)$ и $\operatorname{Cohn}\left(H_{0}, H_{1}\right)$ вместо $\operatorname{Cohn}\left(\left\{H_{0}, H_{1}\right\} \rightarrow\left\{H_{0}, H_{1}\right\}\right)$. Через $L(H)$ будем обозначать пространство всех линейных ограниченных операторов, действующих в пространстве $H$, с обычной операторной нормой.

Оказалось, что давно возникший вопрос о совпадении $S_{1}\left(H_{0}\right) \cap S_{1}\left(H_{1}\right)$ с пространством $\operatorname{Cohn}\left(H_{0}, H_{1}\right)$ когерентно ядерных операторов решается положительно. Это объясняет тот факт, что с точки зрения орбит идеалы $S_{1}\left(H_{0}\right) \cap S_{1}\left(H_{1}\right)$ и $\operatorname{Cohn}\left(H_{0}, H_{1}\right)$ неразличимы (см. [1]). Соответствующая теорема и составляет п. 1. В п. 2 получена формула для $K$-функционала в паре алгебр линейных операторов и ее следствия. В п. 3 рассмотрены интерполяционные теоремы, которые вытекают из совпадения ядерных и когерентно ядерных операторов.

1. Основная теорема. Основные факты, обеспечивающие совпадение ядерных и когерентно ядерных операторов в гильбертовых парах, известны сравнительно давно. K сожалению, они не были своевременно интерпретированы в таком контексте.

Теорема 1. Пусть $\bar{H}=\left\{H_{0}, H_{1}\right\}-$ произвольная регулярная пара гильбертовых пространств, т.е. пересечение $H_{0} \cap H_{1}$ плотно в $H_{0}$ и в $H_{1}$. Пространство ядерных операторов в паре $\bar{H}$ совпадает с пространством когерентно ядерных операторов.

ДокаЗАТЕЛЬСтво. Пусть $U \in S_{1}\left(H_{0}\right) \cap S_{1}\left(H_{1}\right)$. Хорошо известно, что в этом случае оператор $U: H_{0} \rightarrow H_{0}$ представим в виде произведения операторов $V_{0}: H_{0} \rightarrow F_{0}$ и $W_{0}: F_{0} \rightarrow H_{0}$, где $F_{0}$ - гильбертово пространство, а $V_{0} \in S_{2}\left(H_{0} \rightarrow F_{0}\right), W_{0} \in S_{2}\left(F_{0} \rightarrow H_{0}\right)$. При этом $\|U\|_{S_{1}\left(H_{0}\right)}=\left\|W_{0}\right\|_{S_{2}\left(F_{0} \rightarrow H_{0}\right)}\left\|V_{0}\right\|_{S_{2}\left(H_{0} \rightarrow F_{0}\right)}$. То же самое справедливо и для оператора $U \in S_{1}\left(H_{1}\right)$. Очевидно, операторы $W_{0}$ и $W_{1}$ можно считать мономорфизмами, и рассматривать в качестве вложений пространств $F_{0}$ и $F_{1}$ в $H_{0}+H_{1}$. Следовательно, эти пространства образуют гильбертову пару. Заметим, что эту пару можно считать регулярной. Операторы $V_{0}, V_{1}$ задают отображение пары $\left\{H_{0}, H_{1}\right\}$ в паpy $\left\{F_{0}, F_{1}\right\}$, а операторы $W_{0}, W_{1}$ - отображение пары $\left\{F_{0}, F_{1}\right\}$ в пару $\left\{H_{0}, H_{1}\right\}$ (подробнее см. в [1] или [2]). Таким образом, существуют пара гильбертовых пространств $\bar{F}=\left\{F_{0}, F_{1}\right\}$ и операторы $U_{1}: \bar{H} \rightarrow \bar{F}$ и $U_{2}: \bar{F} \rightarrow \bar{H}$ такие, что $U_{1} \in S_{2}\left(H_{0} \rightarrow F_{0}\right) \cap$ $S_{2}\left(H_{1} \rightarrow F_{1}\right), U_{2} \in S_{2}\left(F_{0} \rightarrow H_{0}\right) \cap S_{2}\left(F_{1} \rightarrow H_{1}\right)$ и

$$
\begin{gathered}
U=U_{2} U_{1} \\
\|U\|_{S_{1}\left(H_{i}\right)}=\left\|U_{1}\right\|_{S_{2}\left(H_{i} \rightarrow F_{i}\right)}\left\|U_{2}\right\|_{S_{2}\left(F_{i} \rightarrow H_{i}\right)}, \quad i=0,1 .
\end{gathered}
$$

Любая регулярная гильбертова пара $\bar{F}$ изоморфна паревесовых пространств $\left\{\ell_{2}, \ell_{2}(\omega)\right\}$ (см. [1]). Обозначим через $\left\{e_{j}\right\}_{j \in \mathbb{J}}$ стандартный базис в соответствующем $\ell_{2}$ и положим $y_{j}=U_{2}\left(e_{j}\right), x_{j}^{*}=U_{1}^{*}\left(e_{j}\right)$. Тогда из (1) следует, что

$$
U=\sum_{j \in \mathbb{J}} y_{j} \otimes x_{j}^{*}
$$

В самом деле,

$$
U_{1}=\sum_{k \in \mathbb{J}} e_{k} \otimes x_{k}^{*}, \quad U_{2}=\sum_{j \in \mathbb{I}} y_{j} \otimes e_{j}
$$


поэтому

$$
U_{2} U_{1}=\left(\sum_{j \in \mathbb{J}} y_{j} \otimes e_{j}\right)\left(\sum_{k \in \mathbb{J}} e_{k} \otimes x_{k}^{*}\right)=\sum_{k, j \in \mathbb{J}}\left(e_{j}, e_{k}\right) y_{j} \otimes x_{k}^{*}=\sum_{j \in \mathbb{J}} y_{j} \otimes x_{j}^{*} .
$$

Напомним, что если фиксировать произвольньй ортонормированньй базис $\left\{e_{j}\right\}_{j \in \mathbb{J}}$ в гильбертовом пространстве $H$, то

$$
\|T\|_{S_{2}(H \rightarrow G)}=\left(\sum_{j \in \mathbb{J}}\left\|T\left(e_{j}\right)\right\|_{G}^{2}\right)^{1 / 2} .
$$

Поэтому

$$
\begin{aligned}
\left(\sum_{j \in \mathbb{J}}\left\|x_{j}^{*}\right\|_{H_{0}^{*}}\left\|y_{j}\right\|_{H_{0}}\right)^{2} & \leqslant\left(\sum_{j \in \mathbb{J}}\left\|y_{j}\right\|_{H_{0}}^{2}\right)\left(\sum_{j \in \mathbb{J}}\left\|x_{j}^{*}\right\|_{H_{0}^{*}}^{2}\right) \\
& =\left\|U_{1}\right\|_{S_{2}\left(H_{0} \rightarrow F_{0}\right)}^{2}\left\|U_{2}\right\|_{S_{2}\left(F_{0} \rightarrow H_{0}\right)}^{2}<\infty
\end{aligned}
$$

и

$$
\begin{aligned}
\left(\sum_{j \in \mathbb{J}}\left\|x_{j}^{*}\right\|_{H_{1}^{*}}\left\|y_{j}\right\|_{H_{1}}\right)^{2} & =\left(\sum_{j \in \mathbb{J}}\left(\omega_{j}^{1 / 2}\left\|x_{j}^{*}\right\|_{H_{1}^{*}}\right)\left(\omega_{j}^{-1 / 2}\left\|y_{j}\right\|_{H_{1}}\right)\right)^{2} \\
& \leqslant\left(\sum_{j \in \mathbb{J}} \omega_{j}\left\|x_{j}^{*}\right\|_{H_{1}^{*}}^{2}\right)\left(\sum_{j \in \mathbb{J}} \omega_{j}^{-1}\left\|y_{j}\right\|_{H_{1}}^{2}\right) \\
& =\left\|U_{1}\right\|_{S_{2}\left(H_{1} \rightarrow F_{1}\right)}^{2}\left\|U_{2}\right\|_{S_{2}\left(F_{1} \rightarrow H_{1}\right)}^{2}<\infty .
\end{aligned}
$$

Теорема доказана.

Заметим, что для банаховых пар утверждение, подобное теореме 1 , вообще говоря, неверно (см. [1], [2]).

2. Вычисление $K$-функционала для банаховой пары $\left\{L\left(H_{0}\right), L\left(H_{1}\right)\right\}$. Напомним, что для любой банаховой пары $\bar{X}=\left\{X_{0}, X_{1}\right\}$ определен $K$-функционал Петре на пространстве $X_{0}+X_{1}$ :

$$
K(s, t, x ; \bar{X})=\inf _{x=x_{0}+x_{1}} s\left\|x_{0}\right\|_{X_{0}}+t\left\|x_{1}\right\|_{X_{1}}, \quad s, t>0 .
$$

Пусть $\left\{H_{0}, H_{1}\right\}$ - пара гильбертовых пространств. Вновь будем предполагать, что эта пара регулярна.

Очевидно, что любой линейньй ограниченный оператор $T: H_{0} \rightarrow H_{0}$ действует непрерьвно из $H_{0} \cap H_{1}$ в $H_{0}+H_{1}$, т.е. имеет место непрерывное вложение $L\left(H_{0}\right) \subset$ $L\left(H_{0} \cap H_{1} \rightarrow H_{0}+H_{1}\right)$. Аналогичноевложение справедливо и для пространства $L\left(H_{1}\right)$. Поэтому алгебры операторов $L\left(H_{0}\right)$ и $L\left(H_{1}\right)$ образуют банахову пару. Теорема 1 позволяет описать сумму пространств этой пары и вычислить $K$-функционал.

Teорема 2. Onepamop $U \in L\left(H_{0} \cap H_{1} \rightarrow H_{0}+H_{1}\right)$ принадлежит $L\left(H_{0}\right)+L\left(H_{1}\right)$ тогда и только тогда, когда

$$
\sup _{y \in H_{0} \cap H_{1}} K\left(\frac{1}{\|y\|_{H_{0}}}, \frac{1}{\|y\|_{H_{1}}}, U(y) ;\left\{H_{0}, H_{1}\right\}\right)<\infty
$$

при этом

$$
K\left(s, t, U ;\left\{L\left(H_{0}\right), L\left(H_{1}\right)\right\}\right) \asymp \sup _{y \in H_{0} \cap H_{1}} K\left(\frac{s}{\|y\|_{H_{0}}}, \frac{t}{\|y\|_{H_{1}}}, U(y) ;\left\{H_{0}, H_{1}\right\}\right) .
$$


ДокАЗАТЕЛЬСтво. Пространство $L(H)$ является сопряженньм к пространству ядерных операторов $S_{1}(H)$. Двойственность $L(H)$ и $S_{1}(H)$ может быть задана формулой

$$
\langle U, T\rangle=\operatorname{tr}(U T) .
$$

Покажем, что пара $\left\{L\left(H_{0}\right), L\left(H_{1}\right)\right\}$ является сопряженной к паре $\left\{S_{1}\left(H_{0}\right), S_{1}\left(H_{1}\right)\right\}$. Действительно, $S_{1}\left(H_{0}+H_{1} \rightarrow H_{0} \cap H_{1}\right)$ плотно вложено в $S_{1}\left(H_{0}\right)$ и в $S_{1}\left(H_{1}\right)$ ввиду регулярности пары $\left\{H_{0}, H_{1}\right\}$. Если воспользоваться двойственностью пространств $S_{1}\left(H_{0}+H_{1} \rightarrow H_{0} \cap H_{1}\right)$ и $L\left(H_{0} \cap H_{1} \rightarrow H_{0}+H_{1}\right)$, задаваемой формулой (3) где $T \in S_{1}\left(H_{0}+H_{1} \rightarrow H_{0} \cap H_{1}\right)$, a $U \in L\left(H_{0} \cap H_{1} \rightarrow H_{0}+H_{1}\right)$, то мы получим, что сопряженное пространство к $S_{1}\left(H_{0}\right)$ отождествлено с $L\left(H_{0}\right)$ в $L\left(H_{0} \cap H_{1} \rightarrow H_{0}+H_{1}\right)$, а сопряженное к $S_{1}\left(H_{1}\right)$ - с $L\left(H_{1}\right)$.

Как известно (см. [3]), для любой регулярной пары $\left\{X_{0}, X_{1}\right\}$ справедливо изометрическое равенство

$$
\left(X_{0} \cap X_{1}\right)^{*} \cong X_{0}^{*}+X_{1}^{*} \text {. }
$$

Применяя (4) к паре $\left\{S_{1}\left(H_{0}\right), S_{1}\left(H_{1}\right)\right\}$, получим, что изометрически

$$
L\left(H_{0}\right)+L\left(H_{1}\right) \cong\left(S_{1}\left(H_{0}\right) \cap S_{1}\left(H_{1}\right)\right)^{*},
$$

т.е. $U \in L\left(H_{0}\right)+L\left(H_{1}\right)$, если $\sup _{T}|\operatorname{tr}(U T)|<\infty$, где точная верхняя грань берется по всем операторам из единичного шара в $S_{1}\left(H_{0}\right) \cap S_{1}\left(H_{1}\right)$. В силу теоремы 1 верхняя грань может быть взята по единичному шару в $\operatorname{Cohn}\left(H_{0}, H_{1}\right)$, которьй является замкнутой вьпуклой оболочкой операторов вида $y \otimes x^{*}$, где $x^{*} \in\left(H_{0}+H_{1}\right)^{*} \cong H_{0}^{*} \cap H_{1}^{*}$ и $y \in H_{0} \cap H_{1}$. Иными словами,

$$
\sup _{x^{*}, y}\left|x^{*}(U y)\right|<\infty
$$

где $\left\|x^{*}\right\|_{H_{0}^{*}}\|y\|_{H_{0}} \leqslant 1,\left\|x^{*}\right\|_{H_{1}^{*}}\|y\|_{H_{1}} \leqslant 1$. Это множество мы обозначим через $\mathscr{R}$.

Таким образом, $U \in L\left(H_{0}\right)+L\left(H_{1}\right)$ эквивалентно

при этом

$$
\sup _{\left\{x^{*}, y\right\} \in \mathscr{R}}\left|x^{*}(U y)\right|<\infty,
$$

с универсальными константами.

$$
\|U\|_{L\left(H_{0}\right)+L\left(H_{1}\right)} \asymp \sup _{\left\{x^{*}, y\right\} \in \mathscr{R}}\left|x^{*}(U y)\right|
$$

Очевидно,

$$
\sup _{\left\{x^{*}, y\right\} \in \mathscr{R}}\left|x^{*}(U y)\right|=\sup _{y \in H_{0} \cap H_{1}} \sup _{\left\|x^{*}\right\|_{H_{0}^{*}} \leqslant 1 /\|y\|_{H_{0}}}\left|x^{*}(U y)\right| .
$$

Как следует из [3], для всех $z \in H_{0}+H_{1}$

поэтому

$$
\begin{aligned}
& \sup _{\left\|x^{*}\right\|_{H_{0}^{*} \leqslant \alpha}}\left|x^{*}(z)\right|=K\left(\alpha, \beta, z ;\left\{H_{0}, H_{1}\right\}\right), \\
& \left\|x^{*}\right\|_{H_{1}^{*}} \leqslant \beta
\end{aligned}
$$

$$
\|U\|_{L\left(H_{0}\right)+L\left(H_{1}\right)} \asymp \sup _{y \in H_{0} \cap H_{1}} K\left(\frac{1}{\|y\|_{H_{0}}}, \frac{1}{\|y\|_{H_{1}}}, U(y) ;\left\{H_{0}, H_{1}\right\}\right) .
$$

Аналогично получаем

$$
\|U\|_{L\left(H_{0} \rightarrow G_{0}\right)+L\left(H_{1} \rightarrow G_{1}\right)} \asymp \sup _{y \in H_{0} \cap H_{1}} K\left(\frac{1}{\|y\|_{H_{0}}}, \frac{1}{\|y\|_{H_{1}}}, U(y) ;\left\{G_{0}, G_{1}\right\}\right),
$$

где $\left\{G_{0}, G_{1}\right\}$ - произвольная пара гильбертовых пространств. Поэтому имеет место $(2)$, где константы эквивалентности универсальны. Теорема доказана. 
СЛЕДСТВИЕ 1. Пусть $a \otimes b^{*}-$ одномерный оператор из $L\left(H_{0} \cap H_{1} \rightarrow H_{0}+H_{1}\right)$, m.e. $a \in H_{0}+H_{1}, b^{*} \in H_{0}^{*}+H_{1}^{*}$. Тогда $a \otimes b^{*} \in L\left(H_{0}\right)+L\left(H_{1}\right)$ әквивалентно

$$
\sup _{s, t>0} K\left(s, t, a ;\left\{H_{0}, H_{1}\right\}\right) K\left(\frac{1}{s}, \frac{1}{t}, b^{*} ;\left\{H_{0}^{*}, H_{1}^{*}\right\}\right)<\infty,
$$

при этом

$$
K\left(s, t, a \otimes b^{*} ;\left\{L\left(H_{0}\right), L\left(H_{1}\right)\right\}\right) \asymp \sup _{u, v>0} K\left(u, v, a ;\left\{H_{0}, H_{1}\right\}\right) K\left(\frac{s}{u}, \frac{t}{v}, b^{*} ;\left\{H_{0}^{*}, H_{1}^{*}\right\}\right) .
$$

ДоКАЗАТЕЛЬСТво. В силу теоремы $2 a \otimes b^{*} \in L\left(H_{0}\right)+L\left(H_{1}\right)$, если

$$
\sup _{y \in H_{0} \cap H_{1}} K\left(\frac{1}{\|y\|_{H_{0}}}, \frac{1}{\|y\|_{H_{1}}}, a ;\left\{H_{0}, H_{1}\right\}\right)\left|b^{*}(y)\right|<\infty .
$$

Очевидно,

$$
K\left(s, t, a ;\left\{H_{0}, H_{1}\right\}\right)=\sup _{u, v>0} \min \left\{\frac{s}{u}, \frac{t}{v}\right\} K\left(u, v, a ;\left\{H_{0}, H_{1}\right\}\right)
$$

Поэтому левая часть (6) равна

$$
\begin{aligned}
& \sup _{y \in H_{0} \cap H_{1}} \sup _{u, v>0} \min \left\{\frac{1}{u\|y\|_{H_{0}}}, \frac{1}{v\|y\|_{H_{1}}}\right\} K\left(u, v, a ;\left\{H_{0}, H_{1}\right\}\right)\left|b^{*}(y)\right| \\
& =\sup _{u, v>0} K\left(u, v, a ;\left\{H_{0}, H_{1}\right\}\right) \sup _{y \in H_{0} \cap H_{1}} \frac{\left|b^{*}(y)\right|}{\max \left\{u\|y\|_{H_{0}}, v\|y\|_{H_{1}}\right\}} \\
& =\sup _{u, v>0} K\left(u, v, a ;\left\{H_{0}, H_{1}\right\}\right) K\left(\frac{1}{u}, \frac{1}{v}, b^{*} ;\left\{H_{0}^{*}, H_{1}^{*}\right\}\right) .
\end{aligned}
$$

Аналогично получается и (5). Следствие доказано.

Назовем функцию

$$
f(s, t)=\sup _{u, v>0} f_{1}(u, v) f_{2}\left(\frac{s}{u}, \frac{t}{v}\right)
$$

идемпотентной сверткой функций $f_{1}$ и $f_{2}$. Таким образом, $K$-функционал тензорного произведения $a \otimes b^{*}$ равен свертке $K$-функционалов сомножителей.

Заметим, что если обозначим через $a^{\star}$ элемент из $H_{0}^{*}+H_{1}^{*}$ такой, что

$$
K\left(u, v, a^{\star} ;\left\{H_{0}^{*}, H_{1}^{*}\right\}\right) \asymp K^{\star}\left(u, v, a ;\left\{H_{0}, H_{1}\right\}\right),
$$

где $\varphi^{\star}(u, v)=1 / \varphi(1 / u, 1 / v)$, то получим, что

$$
K\left(1, t, a \otimes a^{\star} ;\left\{L\left(H_{0}\right), L\left(H_{1}\right)\right\}\right) \asymp \rho_{K}(t),
$$

где $\rho_{K}(t)$ - функция растяжения $K\left(1, v, a ;\left\{H_{0}, H_{1}\right\}\right)$ (ср. с аналогичной формулой в [4], где вместо пары $\left\{H_{0}, H_{1}\right\}$ рассматривается пара $\left.\left\{\ell_{1}, \ell_{\infty}\right\}\right)$.

Формула (2) для $K$-функционала приводит к прямому доказательству равенства из [5]

$$
\left(L\left(H_{0}\right), L\left(H_{1}\right)\right)_{\theta, \infty}=L\left(\left(H_{0}, H_{1}\right)_{\theta, 1} \rightarrow\left(H_{0}, H_{1}\right)_{\theta, \infty}\right) .
$$


В самом деле, по определению $U \in\left(L\left(H_{0}\right), L\left(H_{1}\right)\right)_{\theta, \infty}$ означает, что

$$
\sup _{t>0} \frac{K\left(1, t, U ;\left\{L\left(H_{0}\right), L\left(H_{1}\right)\right\}\right)}{t^{\theta}}<\infty \text {. }
$$

Следовательно,

$$
\begin{aligned}
\sup _{t>0} & \sup _{y \in H_{0} \cap H_{1}} \frac{K\left(1 /\|y\|_{H_{0}}, t /\|y\|_{H_{1}}, U(y) ;\left\{H_{0}, H_{1}\right\}\right)}{t^{\theta}} \\
= & \sup _{y \in H_{0} \cap H_{1}} \sup _{t>0} \frac{K\left(1 /\|y\|_{H_{0}}, t /\|y\|_{H_{1}}, U(y) ;\left\{H_{0}, H_{1}\right\}\right)}{t^{\theta}} \\
= & \sup _{y \in H_{0} \cap H_{1}} \frac{\|U(y)\|_{\left(H_{0}, H_{1}\right)_{\theta, \infty}}}{\|y\|_{H_{0}}^{1-\theta}\|y\|_{H_{1}}^{\theta}}<\infty,
\end{aligned}
$$

т.е.

$$
\|U(y)\|_{\left(H_{0}, H_{1}\right)_{\theta, \infty}} \leqslant C\|y\|_{H_{0}}^{1-\theta}\|y\|_{H_{1}}^{\theta}
$$

для некоторой константы $C$ и всех $y \in H_{0} \cap H_{1}$, что эквивалентно ограниченности $U$ из $\left(H_{0}, H_{1}\right)_{\theta, 1}$ в $\left(H_{0}, H_{1}\right)_{\theta, \infty}$.

3. Интерполяция ядерных операторов. Тот факт, что ядерные операторы, действующие в паре гильбертовых пространств, оказываются когерентно ядерными, позволяет усилить интерполяционные теоремы для таких операторов (ср. с [5]).

ТЕорема 3. Если любой оператор $T \in S_{1}\left(H_{0}\right) \cap S_{1}\left(H_{1}\right)$ ограниченно действует из промехуточного пространства $E$ в промехуточное пространство $F$, то оператор $T$ является ядерным из $Е$ в $F$.

ДокАЗАТЕЛЬСТво. Из условия теоремы и принципа равномерной ограниченности следует, что для любого одномерного оператора $y \otimes x^{*} \in S_{1}\left(H_{0}\right) \cap S_{1}\left(H_{1}\right)$

$$
\left\|x^{*}\right\|_{E^{*}}\|y\|_{F} \leqslant C \max \left\{\left\|x^{*}\right\|_{H_{0}^{*}}\|y\|_{H_{0}},\left\|x^{*}\right\|_{H_{1}^{*}}\|y\|_{H_{1}}\right\} .
$$

Поэтому, если $T \in \operatorname{Cohn}\left(H_{0}, H_{1}\right)$, то существуют $x_{j}^{*} \in\left(H_{0}+H_{1}\right)^{*}=H_{0}^{*} \cap H_{1}^{*}$ и $y_{j} \in H_{0} \cap H_{1}$ такие, что

$$
T=\sum_{j \in \mathbb{Z}} y_{j} \otimes x_{j}^{*}
$$

и

$$
\sum_{j \in \mathbb{Z}}\left\|x_{j}^{*}\right\|_{E^{*}}\left\|y_{j}\right\|_{F} \leqslant 2 C \max _{i=0,1} \sum_{j \in \mathbb{Z}}\left\|x_{j}^{*}\right\|_{H_{i}^{*}}\left\|y_{j}\right\|_{H_{i}}<\infty
$$

Теорема доказана.

В [1] показано, что если $T \in S_{1}\left(H_{0}\right) \cap S_{1}\left(H_{1}\right)$, то оператор $T$ непрерьвно действует из обобщенного пространства Марцинкевича

$$
M_{\varphi}\left(H_{0}, H_{1}\right)=\left\{x \in H_{0}+H_{1}: \sup _{s, t>0} \frac{K\left(s, t, x ;\left\{H_{0}, H_{1}\right\}\right)}{\varphi(s, t)}<\infty\right\}
$$

в обобщенное пространство Лоренща

$$
\Lambda_{\varphi}\left(H_{0}, H_{1}\right)=\left\{x \in H_{0}+H_{1}: x=\sum_{j \in \mathbb{Z}} x_{j}, \sum_{j \in \mathbb{Z}} \varphi^{\star}\left(\left\|x_{j}\right\|_{H_{0}},\left\|x_{j}\right\|_{H_{1}}\right)<\infty\right\} .
$$

Таким образом, справедливо 
СлЕДСТВИЕ 2. Если $T \in S_{1}\left(H_{0}\right) \cap S_{1}\left(H_{1}\right)$, әде $\left\{H_{0}, H_{1}\right\}$ - nара гильбертовыX пространств таких, что $H_{0} \cap H_{1}$ плотно в $H_{0}$ и в $H_{1}$, то оператор $T$ ядерный из $M_{\varphi}\left(H_{0}, H_{1}\right)$ в $\Lambda_{\varphi}\left(H_{0}, H_{1}\right)$.

Значит, если $T \in S_{1}\left(H_{0}\right) \cap S_{1}\left(H_{1}\right)$, то $T$ будет ядерным в любом пространстве $E$, удовлетворяющем условиям

$$
\Lambda_{\varphi}\left(H_{0}, H_{1}\right) \subset E \subset M_{\varphi}\left(H_{0}, H_{1}\right) .
$$

Как известно (см. [1] или [6]), условия (7) эквивалентны

$$
\|x\|_{E} \leqslant \varphi^{\star}\left(\|x\|_{H_{0}},\|x\|_{H_{1}}\right), \quad\|f\|_{E^{*}} \leqslant \varphi\left(\|f\|_{H_{0}},\|f\|_{H_{1}}\right)
$$

для всех $x \in H_{0} \cap H_{1}$ и $f \in\left(H_{0}+H_{1}\right)^{*}$.

Заметим, что следствие 2 обобщает результаты из [5], где показано, что $T$ - ядерньй оператор из пространства $\left(H_{0}, H_{1}\right)_{\theta, \infty}$ в пространство $\left(H_{0}, H_{1}\right)_{\theta, 1}$.

\section{СПИСОК ЦИТИРОВАННОЙ ЛИТЕРАТУРЫ}

[1] Ovchinnikov V. I. The method of orbits in interpolation theory // Math. Rep. 1984. V. 1. № 2. P. 349-515.

[2] Овчинников В. И. Интерполяция операторов классов $S_{p}$ в гильбертовых парах // Матем. заметки. 1980. Т. 27. №2. С. 273-282.

[3] Bergh J., Löfström J. Interpolation Spaces. An Introduction. Berlin-Heidelberg-New York: Springer, 1976.

[4] Овчинников В.И. Вычисление $K$-функционала для пар пространств матриц // Матем. заметки. 1994. Т. 55. № 5. С. 98-109.

[5] Овчинников В. И. Интерполяция в симметрично-нормированных идеалах операторов, действующих в различных гильбертовых пространствах // Функцион. анализ и его прилож. 1994. Т. 28. № 3. С. $80-82$.

[6] Дмитриев В. И., Крейн С. Г., Овчинников В. И. Основы теории интерполяции линейных операторов // Геометрия линейных пространств и теория операторов. Ярославль, 1977. C. $31-74$. 